Lucie LEDOuX, «Cixous : autour de l'archive », @ nalyses, printemps 2007

\title{
Lucie LEDOUX
}

\section{Cixous : autour de l'archive}

À la suite de la donation par Hélène Cixous de l'ensemble de ses manuscrits et archives personnels, il s'est tenu à la Bibliothèque nationale de France, en mai 2003, un colloque intitulé Genèses Généalogies Genres. Le sous-titre, plus conventionnel, situait le lecteur : Autour de l'cuvre d'Hélène Cixous.

La rencontre a eu lieu, comme l'explique Jean-Noël Jeanneney, «pour célébrer sa personne et son œuvre » (p. 13). La célébrer elle : Hélène Cixous. Celle dont l'œuvre est empreinte de biographique, comme le note René de Cettatty: « la mort précoce du père, la nationalité allemande de la mère, l'identité juive, le frère qui accompagne la vie entière, la découverte littéraire » (p. 326). Pour cette célébration, plusieurs moyens seront utilisés: une mise en lecture de La Ville parjure par les comédiens du Théâtre du Soleil, suivie d'une discussion; la lecture d'une lettre personnelle adressée à la femme sauvage par Adonis, ami de Cixous; une table ronde (avec Bertrand Leclair, René de Ceccatty et Tiphaine Samoyault), animée par Mireille Calle-Gruber, suivie d'un entretien avec l'auteure du Jour où je n'étais pas là; l'extrait d'un roman inédit en français signé Alia Mamdouh et dont le titre original est Al Mabbubat (Les bien-aimées) et, bien sûr, les vingt et une communications (vingt-deux en comptant celle de Cixous, puisque la principale intéressée propose un texte intitulé «Le Livre que je n’écris pas »).

L'avant-propos est signé par les directrices de l'ouvrage : Marie Odile Germain et Mireille Calle-Gruber. C'est dans cette section que l’originalité du recueil est soulignée: celle d'offrir un éventail de contributions de « romanciers et poètes, venus de France, d'Amérique, du Maghreb ou d'Orient »(p. 10). Amitiés, multiculturalisme et littérature. Voilà quelques thèmes qui relient ces différentes 
Lucie LEDOuX, «Cixous : autour de l'archive », @ nalyses, printemps 2007

allocutions. Un mot à la mémoire d'Annie Leclerc, décédée en novembre 2006, est annexé à la fin de cet avant-propos.

Le texte de bienvenue, signé Mireille Calle-Gruber et intitulé «Le Legs d'Hélène» donnera le ton, qui sera repris par presque tous les participants. D'abord, on se réclame de Derrida. Ici, ses mots figurent en exergue : «On peut seulement rêver de donner/On ne peut donner qu'en rêvant/C'est un rêve » (p. 15). Des mots qui "appellent la grâce » (p. 15), selon Calle-Gruber, qui ne parle pas des archives de Cixous, mais plutôt de « ses peaux les plus intimes » (p. 15); pour qui la BNF est " la magique Maison des Mémoires », un des "Toits des Mémoires, comme on dit pour le Tibet Toit du Monde ", lieu sacré donc où les participants sont « engagés dans un travail du don » (p. 16) et où ils se rencontrent afin de produire une "prière à plusieurs mains » (p. 16).

Puis vient un dialogue entre Derrida et Cixous publié en 2004 par Le Magazine littéraire et partiellement reproduit ici pour servir de pont entre les textes d'ouverture et les communications. Symboliquement, il témoigne aussi d'une longue et profonde amitié. Derrida disait de ce dialogue qu'il était un "exemple récent de tous les "partages" que nous pouvions seulement évoquer ici » (p. 21). Ce n'est qu'après les mots de l'ami sur le partage ("Jacques Derrida votre ami, votre complice », disait Jean-Noël Jeanneney, p. 15) que débute véritablement cet ouvrage.

Défilent alors les vingt-deux textes des communications, dont on retiendra surtout les noms de Charles Malamoud, Eberhard Gruber, Jacques Aubert, Gil Anidjar, Marie-Claire Blais, Béatrice Didier, Ariane Mnouchkine, René Major, Frédéric Regard, Tom Conley, JeanClaude Coquet, Jean-Jacques Lemêtre et Michèle GendreauMassaloux. Qui sont ces participants? Quels sont les liens qui les unissent à Cixous? Ont-ils consacré des années de recherche à son œuvre? Sont-ils plutôt des amis? Des lecteurs? Nous n'en savons rien puisqu'aucune notice biobibliographique n'accompagne les textes. L'effet produit est celui d'une rencontre entre intimes. C'est d'ailleurs probablement le but recherché : l'impression d'être en famille ou entre 
amis plutôt qu'avec des intellectuels et d'éminents chercheurs (certains sont pourtant des spécialistes de l'œuvre cixousienne). Chaque intervenant s'intéresse à une œuvre de l'auteure et, sans jamais être couverts en entier, ses écrits (fictions, essais et théâtre) sont toutefois bien représentés. Tour à tour, lecteurs, amis, spécialistes, analystes et universitaires viennent parler d'Hélène Cixous, parler à Hélène, parler d'une ou de plusieurs de ses œuvres, dont on trouve à la fin du volume une utile table de références.

Puisqu'il s'agit d'une rencontre organisée lors d'une donation de manuscrits, la question des archives revient dans presque toutes les communications. En témoignent les quelques titres suivants: «Osnabrück: les commencements du commencement. Un premier regard sur les manuscrits d'Hélène Cixous» (Daniel Ferrer); «Mémoire de manuscrits» (Marie Odile Germain); "Témoigner à distance » (Abdelkébir Khatibi); quant au texte de Ginette Michaud, «Ombilic (l'œuvre à l'insu de l'archive) », il s'intéresse à l'archive, mais aussi à la naissance et aux commencements. Fait intéressant: sont reproduits quelques textes écrits à la main par Cixous. Nous y voyons des manuscrits raturés, d'autres auxquels l'auteure a fait quelques ajouts, des " $\grave{A}$ faire », ainsi qu'un amoncellement de notes. Ce thème des débuts, de la naissance et de l'origine est indissociable de l'archive certes, et constitue la matière fondatrice de l'œuvre cixousienne.

En lisant cet Autour de (qui devient rapidement un Hommage a), un autre détail que l'on remarque rapidement est la façon dont presque tous les participants semblent naturellement adopter le même style. Ton inspiré par Hélène Cixous, par son œuvre, par sa présence ou simple hasard? Ajoutons que cette particularité n'enlève rien au plaisir de lecture. D'ailleurs, il est certainement plus question de plaisir que d'utilité : il ne s'agit pas ici d'un livre utile dans la mesure où, travaillant sur l'œuvre de Cixous, on pourrait y trouver quantité d'informations importantes produites par d'éminents spécialistes de l'œuvre cixousienne. Il s'agit plutôt du plaisir de retrouver le ton, l'univers, les mots de Cixoux, même s'il est effectivement question d'experts, d'amis-chercheurs ou d'admirateurs-spécialistes de cette œuvre: notons toutefois que le type de lecture linéaire que nous 
adoptons ici à des fins de recension est plutôt rare lorsqu'il s'agit de la publication des actes d'un colloque, le lecteur préférant en faire habituellement une lecture aléatoire ou motivée par un intérêt précis et parfois exclusif.

À la suite de ces vingt-deux communications toujours très courtes (donnant à nouveau l'impression d'une discussion entre intimes), est reproduite une lettre personnelle écrite par Adonis, ami de l'écrivaine, et qui déclare solennellement : «Oui, j’aime te lire Hélène, j'aime aller vers tes déluges » (p. 312). Une table ronde, coordonnée par Mireille Calle-Gruber et intitulée "Scènes de lecture ", prend place après ce vibrant hommage. Cette table ronde est le seul moment où les participants nomment leur fonction: éditeur, traducteur, écrivain, critique littéraire. Ils discutent de la lecture (de leur lecture de Cixous), de la critique (de leur critique de l'œuvre de Cixous). Cette section se termine sur les mots de Mireille Calle-Gruber : « on arrête sur ce mot: miracle » (p. 352). En final est reproduit un extrait du roman Les bienaimées, de Alia Mamdouh, auteure iraquienne régulièrement censurée en raison des thèmes qu'elle aborde dans ses livres, notamment la lutte pour les droits des femmes. Ce texte est reproduit ici en guise de conclusion. Il semble s'adresser directement à la principale intéressée : "Nous étions plantés là, attendant ton arrivée. Quels mots allais-je prononcer, balbutiante et trébuchante, retenant fermement les survivants pour les empêcher de quitter les traits de mon visage? » (p. 354). Car effectivement, c'est bien de cela qu'il s'agit dans ce colloque : d'admiration, de mots prononcés, de fragilités, de spectres. Toujours dans le texte d'Alia Mamdouh, à propos de la protagoniste qu'on attend fébrilement, il est dit d'elle qu'« elle avait lancé son fameux I am not a feminist. C'était il y a des années. À présent, elle avait établi sa nouvelle théorie sur l'écriture féminine, ou plutôt la féminisation de l'écriture. » (p. 355) Ce qui nous rappelle à quel point l'aspect féministe du parcours de Cixous est, dans ce colloque, totalement oblitéré. À part la petite remarque en début d'ouvrage, voici les deux seuls moments où il est question de cette posture pourtant très importante dans l'œuvre et la vie d'Hélène Cixous : un mot rapide lors du texte d'ouverture et une allusion lors de la 
Lucie LEDOuX, «Cixous : autour de l'archive », @ nalyses, printemps 2007

conclusion. C'est très peu, compte tenu de son engagement dans ce domaine.

En terminant cette trop rapide recension, ajoutons que presque tous les participants rendent compte de l'œuvre de Cixous dans une langue et un style tragiques, donnant davantage une impression de cérémonie funèbre que de célébration. Dans le même sens, l'épigraphe en l'honneur d'Annie Leclerc ne peut qu'en appeler une autre, celle de Jacques Derrida, qui sera présent (voire vivant) tout au long du volume. Ami de Cixous, spécialiste de son œuvre, il sera nommé ou cité à de nombreuses reprises par tous les intervenants. En somme, voilà un livre sur l'admiration, la considération, l'hommage et la déférence davantage qu'une étude savante ou un ouvrage d'analyse littéraire. Cette publication des actes de colloque tourne bel et bien autour de l'œuvre de Cixous. Ce qui est célébré cependant, c'est la filiation, l'amitié, le lignage : "je suis une descendante de morts. [...] Mes morts vivent dedans moi », dit Hélène Cixous (p. 238). Bien sûr, ce sont aussi les mots, la parole et la littérature qui sont encensés : «Oui, disait Jacques Derrida, au commencement il y a le mot. » (p. 21)

Référence : Mireille Calle-Gruber et Marie Odile Germain (dir.), Genèses Généalogies Genres. Autour de l'œuvre d'Hélène Cixous, Paris, Éditions Galilée/Bibliothèque nationale de France, coll. «Lignes fictives », 2006, $369 \mathrm{p}$. 